\title{
Zum Tode von Gerhard Brandt (3.2.1929-25.11.1987)
}

Alle, die ihn kannten, waren tief betroffen, als sie von seinem Tode erfuhren; für die, die mit ihm befreundet waren, war es ein Schock. Daß er Hand an sich legte und sich das Leben nahm, war soweit wir es wissen können - keine plötzliche Kurzschlußhandlung; es hatte sich schon seit längerem vorbereitet. Freilich ahnte keiner, auch nicht die ihm am nächsten waren, daß er sich in einer ausweglosen Situation empfand. $\mathrm{DaB}$ er dies auch vor seinen nächsten Kollegen abzuschirmen vermochte, gehört zu den ungelösten Problemen, an denen er litt. Die Reserviertheit und Distanziertheit, die für ihn im alltäglichen Umgang so charakteristisch waren, verbargen eine fragile psychische Konstitution. Innere Sicherheit fand er in der rastlosen wissenschaftlichen Arbeit, in der Sorgfalt und Akribie, mit der er Forschung und Lehre betrieb. Den Stand der Forschung und Theoriebildung bis in die entlegensten Bereiche der eigenen Disziplin zu überschauen, war für ihn selbstverständliche Verpflichtung - ebenso wie die auf Intentionen und Anspruch der Kritischen Theorie. Seine wissenschaftlichen Leistungen - das wird jetzt nach seinem Tode deutlich - wurden einem stets gefährdeten Leben abgezwungen, mit einem Aufwand an psychischer Energie, der nur schwer zu ermessen ist. Schließlich seine nahezu grenzenlose Hilfsbereitschaft und nie versagende Loyalität gegenüber Kollegen und Studierenden, die er wie kein Zweiter praktizierte. Nie demonstrierte er Überlegenheit - eine im Wissenschaftsbetrieb seltene Tugend. Er nahm vieles an psychischen Kosten auf sich, die im Universitäts- und Forschungsbetrieb in der Regel auf Konkurrenten und Jüngere abgewälzt werden. Was auch immer daran seinem Charakter zuzuschreiben sein mag allen, die mit ihm zu tun hatten, ist das unverdient zugute gekommen.

1929 geboren, gehörte Gerhard Brandt zu der Generation, für die Faschismus, Krieg und Kriegsende noch bewußte und prägende Erfahrung war und die sie, oft auf Umwegen, zur Soziologie führte. Auf das Abitur (1948) folgten zunächst eine Handwerkslehre als Zimmermann, Mitarbeit in der Gewerkschaftsjugend in Hannover, dann ein Praktikum an einem Jugendamt in Berlin. Nach einem einjährigen Studienaufenthalt in den USA am Utica College der Syracuse University begann er das Soziologiestudium 1953, zunächst für zwei Semester in Göttingen, ab 1954 dann an der Universität Frankfurt. Gerhard Brandt gehörte zur ersten Generation von Studierenden, die bei Horkheimer und Adorno am Institut für Sozialforschung ihre Soziologieausbildung erhielten. Theoretisch begabt und politisch motiviert, trugen sie nicht wenig dazu bei, den Seminaren am Institut jenen unverwechselbaren intellektuellen Stil, ja Glanz zu verleihen. Wir Jüngeren haben dabei und von ihnen viel gelernt. Schon vor seinem Diplomabschluß (1958) arbeitete Gerhard Brandt als wissenschaftliche Hilfskraft für ein bescheidenes Salär an mehreren empirischen Untersuchungen des Instituts mit. Wie einige andere der jüngeren Assistenten und älteren Studenten am Institut „entdeckte" Gerhard Brandt in den späten Fünfzigern die von Horkheimer und Adorno verleugnete Dimension der Kritik der Politischen Ökonomie: die alte Zeitschrift für Sozialforschung, Neumann, Grossmann, dann Dobb und Sweezy, die „Monthly Review“, die den aktuellen Diskussionsstand der Marxschen Ökonomie repräsentierten. Gerhard Brandt gehörte zu jener Gruppe von Assistenten am Institut, die - in einer nun schon legendären Sitzung - von ihren Lehrern Aufschluß über ihre Stellung zu den theoretischen Positionen der dreißiger Jahre verlangten. Für Gerhard Brandt brachte sie den Abschied aus dem Institut.

Spätestens seit dieser Zeit ging er auf deutliche Distanz zu jener, vor allem auf Ideologiekritik ausgerichteten Version von Kritischer Theorie, wie sie Horkheimer und Adorno vertraten.

Diese prekäre Lage ließ seine psychische Verfassung nicht unberührt. Gestützt und gefördert wurde er in dieser für ihn objektiv wie subjektiv schwierigen Situation von Ludwig von Friedeburg. Mit seiner Hilfe erhielt Gerhard Brandt ein Stipendium für einen einjährigen Studienaufenthalt an der London School of Economics (1960); er vermittelte ihm Forschungsaufträge der Evangelischen Studiengemeinschaft über die Aufgaben der Militärpublizistik und die Rolle der Wirtschaftsverbände bei der Wiederaufrüstung (1961-63). Schließlich wurde er sein Assistent, zunächst an der FU Berlin (1963-66) und seit 1966 wieder an der Universität Frankfurt. 
Die in jenen Jahren entstandene Dissertation „Rüstung und Wirtschaft" (1964) behandelt die keineswegs konvergierenden Interessen von Wirtschaft, Regierung und Militär und ihre spezifische Formung durch Verbandsstrategien und ihren Einfluß auf die Ausbildung militärpolitischer Doktrinen. Auch heute noch ist es von einigem Interesse zu lesen, wie der Druck der Wirtschaftsverbände zu einer weitgehenden Revision der militärischen Planung und zur Übernahme der Abschreckungsstrategie führte. Kennzeichnend für die Promotionsschrift - ebenso auch für die im gleichen Zusammenhang entstandene Schrift zur Militärpublizistik (1966, zusammen mit Ludwig von Friedeburg) - ist ihre Ausrichtung an einer politisch gewendeten Modernisierungstheorie. Gestützt vor allem auf amerikanische Autoren wird das Verhältnis von Politik, Militär und Wirtschaft am Leitfaden einer aufgeklärten, demokratischen Prinzipien verpflichteten Rationalität analysiert. Im Meinungsklima der ausgehenden Adenauerära mit ihren vordemokratischen Traditionsmustern hatte eine solche Argumentationsstrategie eine durchaus kritische Funktion; die Schrift über die Aufgaben der Militärpublizistik war Gutachten für das Bundesverfassungsgericht im „Spiegel“-ProzeB. Die politische Vorsicht, die sich Horkheimer und Adorno in jenen Jahren auferlegten, wurde freilich auch von den Promovenden verlangt.

Die zweite größere Arbeit von Gerhard Brandt, "Gewerkschaftliche Interessenvertretung und sozialer Wandel“ (1975), mit der er sich 1971 an der Universität Frankfurt habilitierte, läßt Motive der Kritischen Theorie schon deutlicher erkennen. Sie behandelt die Periode der Durchsetzung des von Angelernten getragenen „New Unionism“ (1886-1917) und ihre Konflikte mit älteren traditionsreichen Gewerkschaften der „Arbeiteraristokratie" in der britischen Stahlindustrie - eine Arbeit, die weder von den Industriesoziologen noch von den Sozialhistorikern hinreichend zur Kenntnis genommen wurde. Die methodisch und theoretisch sehr reflektierte Analyse stellt einen diskussionswürdigen Versuch dar, mit Hilfe der Kategorien: „normative Handlungsorientierungen“ und „materielle Handlungsbedingungen" und ihres wechselnden Vehältnisses handlungs- und strukturtheoretische Dimensionen zu verknüpfen. Freilich figuriert der Akkumulationsprozeß in der Analyse nur als externe Größe, im Anschluß an Kirchheimer als ,restriktive Bedingung“ gefaßt.

Schon während des Habilitationsverfahrens hatte Gerhard Brandt 1970 eine Lehrstuhlvertretung an der Universität Heidelberg übernommen. Obwohl man ihn dort zu halten suchte, nahm er auf Drängen vieler Kollegen den Ruf der Universität Frankfurt an und kehrte schon 1971 an den Fachbereich Gesellschaftswissenschaft zurück. 1972 übernahm er die Stelle eines Direktors am verwaisten Institut für Sozialforschung. Mit seiner Rückkehr nach Frankfurt hat Gerhard Brandt sich ausdrücklich in die Traditionslinie der Kritischen Theorie gestellt. In zwei programmatischen Texten - „Traditionelle und Kritische Theorie heute" (nicht publiziert. 1971) und „Industrialisierung, Modernisierung, gesellschaftliche Entwicklung" (1972) - hat er ihre Defizite benannt und ein Forschungsprogramm skizziert, das, zentriert auf die Kritik der Politischen Ökonomie und um handlungstheoretische Kategorien erweitert, Antwort auf die Frage geben müsse, „wie und von wem das gesellschaftliche Mehrprodukt appropriiert wird“ und ,von welchen Verhaltensorientierungen sich diejenigen leiten lassen, die über das Mehrprodukt verfügen, und diejenigen, denen es abverlangt und vorenthalten wird“. Die Einlösung dieses Programms einer historisch gerichteten Theorie industriell-kapitalistischer Gesellschaften erhielt seine spezifische Wendung durch die Rezeption der Sohn-Rethelschen Version von Kritischer Theorie am Institut für Sozialforschung.

Gerhard Brandt hat, als er die Direktorenstelle am Institut übernahm, eigene Interessen zurückgestellt und sich in bestehende Forschungs- und Diskussionszusammenhänge eingefügt. Ebenso wie er und seine Arbeit davon geprägt wurden, prägte er die Arbeiten des Instituts in den Siebziger Jahren. Ohne ihn, das wird im Rückblick deutlich, hätten sie schwerlich jene Folgerichtigkeit und innere Konsequenz erhalten, die sie tatsächlich haben. $\mathbf{Z u}$ nennen sind vor allem die an die historische Untersuchung zum Leistungslohn sich anschließenden zwei Computer-Studien mit ihren schrittweisen Revisionen der Sohn-Rethelschen Kategorien und die Sequenz von Untersuchungen über die $\mathrm{Ge}$ werkschaftspolitik, die den Begriff der ,intermediären Gewerkschaft" zu einer empirisch gehaltvollen und theoretisch tragfähigen Kategorie machten. Gerhard Brandt hat diese Forschungen nicht nur angeregt und durch Kritik gefördert, sondern zu ihrem Gelingen auch immer wieder mit eigenen Analysen beigetragen. Darüberhinaus bestand seine spezifische Leistung darin, daß er die Resultate der Forschungsarbeiten stets erneut einer eindringlichen und kritischen Reflexion unterzog. In seiner großen Abhandlung „Ansichten kritischer Sozialforschung 1930-1980“ (1981) hat er 
diese Reflexionen resümiert und Rechenschaft über seine Tätigkeit am Institut abgelegt. Mit guten Gründen kann er behaupten, daß in den Siebziger Jahren ein Forschungsprogramm realisiert wurde, das „die Produktionssphäre als Moment des Reproduktionsprozesses im gegenwärtigen Kapitalismus" ins Zentrum stellte, mithin Motive aus der Frühphase des Instituts wieder aufnahm. Zugleich bezeichnet Gerhard Brandt jedoch das paradoxe Resultat dieses Versuchs: daß nämlich das Theorem von der reellen Subsumtion im Durchgang durch die industriesoziologische Empirie als immanentes Moment kapitalistischer Vergesellschaftung sich erwies und damit die These Horkheimers und Adornos von der Integrationskraft und Selbststabilisierungsfähigkeit des Kapitalismus bestätigt wurde. Was als Schranke reeller Subsumtion in den Forschungen sich zeigte - sei es die Widerständigkeit von Arbeitskraft und ihre prekäre Integration in gesamtgesellschaftliche Steuerungsprozesse, sei es die stoffliche, „Gebrauchswertseite" von Arbeit - ist nicht geeignet, darüber machte sich Gerhard Brandt keine Illusionen, weitergehende politische Hoffnungen zu stützen.

Nicht zu übersehen ist, daß Gerhard Brandt die auf Arbeit und Produktion bezogene Fassung des Subsumtionstheorems zunehmend für revisionsbedürftig, weil in ihrer Reichweite für begrenzt hielt. In seiner weitausholenden Kritik an der deutschen Industriesoziologie, „Marx und die neuere deutsche Industriesoziologie“ (1984), entwickelte er auf die esoterischen Beiträge von Arnason, Ötzel und Bahr zurückgreifend - die These komplementärer oder auch substitutiver „Modi“" kapitalistischer Vergesellschaftung. Einen solchen Modus sah er vor allem in der "immateriellen Produktion“ der „verwissenschaftlichten Technik“, die ungleich nachdrücklicher als materielle Arbeit den Reproduktionsprozeß in den gegenwärtigen kapitalistischen Gesellschaften bestimme. Diesen Gedanken hat er in seinem Beitrag zum industriesoziologischen Technik-Kolloquium (1983) zu entfalten und zu konkretisieren versucht. Von seinem letzten, von ihm angeregten Forschungsprojekt über die „innovativen Subsysteme“ von großen Unternehmen als Konstitutionsbedingungen von verwissenschaftlichter Technik versprach er sich Aufschlüsse über die Tragfähigkeit dieses Gedankens.

Gerhard Brandt war in den letzten Jahren zunehmend davon überzeugt, daß von der Marxschen Theorie allein das Subsumtionstheorem Gültigkeit beanspruchen könne: daß bis auf bloße Reste alle gesellschaftlichen Ressourcen, Arbeit wie Natur, in den Akkumulationsproze $B$ hineingezogen würden. Mit unverkennbarer Sympathie hat er in seinem Beitrag zum Horkheimer-Kolloquium 1985 die skeptischen Motive aus den späten „Notizen“ (1974) aufgenommen. Nur der Verzicht auf „universalistische Theorieentwürfe“ und jedwede Hoffnungen auf einen irgend angebbaren „Sinn in der Geschichte" und im Bewußtsein der historischen Zufälligkeit und Relativität des eigenen theoretischen Denkens könne den Weg frei machen für eine Theorie, die der entfesselten Dynamik der gesellschaftlichen Entwicklung auf die Spur kommt.

Als Gerhard Brandt aus freien Stücken aus dem Institut für Sozialforschung ausschied, geschah dies mit der erklärten Absicht, die gewonnenen Forschungserfahrungen theoretisch aufzuarbeiten. $\mathrm{Daß}$ es dazu nicht kam, hat gewiß viele Gründe. Er betrieb aktiv den Aufbau einer interdisziplinären Arbeitsgruppe für Technikforschung und begann das Projekt über die Konstitutionsbedingungen von Technik. Dazu kamen die Pflichten der akademischen Selbstverwaltung und eines Dekanats am Fachbereich Gesellschaftswissenschaften. Soweit es wir als Außenstehende wissen können, war sein Leben in den letzten Jahren allein von Arbeit bestimmt. Denkbar ist, daß darin sich Züge einer Flucht vor selbst auferlegten theoretischen Verpflichtungen anzeigten: daß es ihm nicht gelang, die Forschungserfahrungen und vielfältigen theoretischen Motive, die er sich aneignete, in Thesen und Theoremen $\mathrm{zu}$ verdichten, die seinen Intentionen und den gesetzten Ansprüchen hätten genügen können. Das hatte gewiß auch mit seiner Charakterkonstitution und ihren spezifischen Problemen zu tun. Unabhängig davon zeigte sich in seinen subjektiven Schwierigkeiten auch das objektive Dilemma der kritischen Gesellschaftstheorie: Wenn der „verschwiegenen“ oder „eingestandenen" Orthodoxie nicht mehr vertraut werden kann, wie kann dann - so die Frage, die alle Arbeiten von Gerhard Brandt anleitete - der Überzeugung, daß der Akkumulationsproze $B$ nach wie vor Zentrum und Triebkraft der gesellschaftlichen Dynamik ist, theoretisch angemessen Ausdruck gegeben werden? $\mathrm{Da}$ er es immer wieder versuchte, durch die Aneignung des Bestandes an soziologischen Theorien wie durch die Systematisierung und theoretische Interpretation von Forschungsresultaten; daß er sich stets erneut Rechenschaft gab über den Stand seiner eigenen Arbeit im Lichte des theoretischen Erbes, dem er sich verpflichtet fühlte, zeugt von einem seltenen, vorbildlichen MaB an intellektueller Redlichkeit. Der Be- 
lastung der selbst auferlegten Ansprüche, so müssen wir vermuten, glaubte er schließlich nicht mehr Stand halten zu können. 